\title{
Element Edge Based Discretization for TCAD Device Simulation
}

\author{
Juan E. Sanchez, Senior Member, IEEE, and Qiusong Chen
}

\begin{abstract}
Technology computer-aided design (TCAD) semiconductor device simulators solve partial differential equations (PDE) using the finite volume method (FVM), or related methods. While this approach has been in use over several decades, its methods continue to be extended, and are still applicable for investigating novel devices. In this paper, we present an element edge based (EEB) FVM discretization approach suitable for capturing vector-field effects. Drawing from a 2D approach in the literature, we have extended this method to $3 \mathrm{D}$. We implemented this method in a TCAD semiconductor device simulator, which uses a generalized PDE (GPDE) approach to simulate devices with the FVM. We describe how our EEB method is compatible with the GPDE approach, allowing the modeling of vector effects using scripting. This method is applied to solve polarization effects in a 3D ferro capacitor, and a 2D ferroelectric field-effect transistor. An example for fielddependent mobility in a 3D MOSFET is also presented.
\end{abstract}

Index Terms_-Device Simulation, FeFET, MOSFET, Semiconductor, TCAD

\section{INTRODUCTION}

$\mathbf{T}$ ECHNOLOGY computer-aided design (TCAD) semiconductor device simulators solve continuum partial differential equations (PDEs) of the drift-diffusion model (DDM) for semiconductors on a discretized mesh [1]. The finite volume method (FVM) is the most widely used approach for assembling the PDEs. In the TCAD device simulation literature, the FVM is referred as finite boxes or control volume [2].

The popularity of the FVM is due to the success of the Scharfetter-Gummel method (SGM) in calculating the electron and hole current densities from exponentially varying carrier densities [3]. It exhibits superior numerical stability to other approaches [1], [4]. While the finite element method (FEM) has some advantages over the FVM [5], [6], the lack of a SGM equivalent often leads to hybrid FVM/FEM approaches for solving the device equations [7]-[9].

Standard simulation models fit well into a node based approach, as volume and surface integration of the PDEs are

The work of $Q$. Chen was supported by Guizhou Provincial Science and Technology Projects (Grant No: QKHJC-ZK[2021]329). (J. E. Sanchez and Q. Chen are co-first authors.)

J. E. Sanchez is with DEVSIM LLC, PO Box 50096, Austin, TX 78763, USA (e-mail: jsanchez@devsim.com)

Q. Chen, is with the Department of Materials Science, Fudan University, 220 Handan Road, Shanghai 200433, China. Q. Chen is also with the School of Physics and Electronic Science, Guizhou Education University, 115 Gaoxin Road, Wudang District, Guiyang, Guizhou, 550018, China. (e-mail: chenqiusong@gmail.com) accounted for in the calculation of quantities at the mesh nodes, and at the edges connecting to adjacent mesh nodes.

Advanced simulation models use vector fields and require information from node quantities off of the edge. For mobility models, this requires knowledge of electric field parallel and normal to the direction of current flow [10]. For the ferroelectric effect, the vector components of electric field and polarization are considered [11], [12]. Nodal approaches have been proposed for evaluation of these models, which consider all adjacent nodes to the node of interest [13], [14]. These approaches consider a constant field over the entire control volume bounded by these nodes.

In contrast, an element edge based (EEB) approach has a control volume bounded by the sub volume along each edge of the element being considered. Laux et al. used a 2D EEB approach to model generalized mobility [10] and impact ionization [15], where vector fields are calculated for each edge of a triangular element. In [16], this discretization was compared with other approaches for impact ionization, and it was found to be the least susceptible to changes in mesh size. This was attributed to the EEB approach using a smaller effective control volume for each element edge.

Generalized PDE (GPDE) simulators use an equation description as input from the user [4], [6], [14], [17]. The goal is to allow the rapid development of new models and applications for the continuum based approach.

Many GPDE simulators do not consider vector effects, and equation assembly is often restricted to node and edge based quantities [4]. Other simulators do not completely model sensitivities of vector effects with respect to nodes off of simulation edges. One group compared 3 TCAD simulation approaches to model the ferroelectric capacitance effect [14]. They report better convergence for methods where more complete model sensitivities are considered, such as ensuring that the electric field is fully coupled with the field dependent polarization model.

In this paper, we describe an EEB approach to modeling vector-field effects in DEVSIM, an open source GPDE semiconductor device simulator [18]. We have implemented the original 2D approach from [10], and have extended it for 3D simulation. To our knowledge, this is the first published extension of the method to 3D for unstructured tetrahedral meshes. In addition, we have provided the EEB method in a Python interface [19]. We believe our approach extends the state of the art by providing a scripting interface to rapidly develop vector-based models with self-consistent model derivatives.

We describe the approach in Section II. In Section III, we 
present simulation examples. We begin with the modeling of hysteresis effects in a ferroelectric capacitor in 3D and in a ferroelectric field effect transistor (FeFET) in 2D. In addition, we present results for transverse electric field effects on mobility in a 3D MOSFET.

\section{Simulator Methods}

\section{A. Equation Assembly}

1) Newton Method: The Newton method requires the evaluation of the model equations, as well as the derivatives with respect to the solution variable [1]. This results in a formulation:

$$
\mathbf{J} \boldsymbol{\Delta} \boldsymbol{x}=-\boldsymbol{F}
$$

where $\mathbf{J}$ is the Jacobian, $\boldsymbol{\Delta} \boldsymbol{x}$ is update to the solution vector, and $\boldsymbol{F}$ is referred to as the right-hand side (RHS) vector. As the method converges at each iteration, $|\boldsymbol{F}| \rightarrow 0$ and $|\boldsymbol{\Delta} \boldsymbol{x}| \rightarrow 0$, to the limits of floating point precision. For TCAD simulation, the nonlinear nature of the PDEs requires a reasonable initial guess, and accurate derivatives, to get convergence.

The PDEs considered in this paper fit in the form

$$
\boldsymbol{F}_{a}: \quad \nabla \cdot \boldsymbol{C}_{a}+\nabla \cdot \boldsymbol{A}_{a}+\boldsymbol{B}_{a}=\mathbf{0}
$$

where $\boldsymbol{C}_{a}$ is an EEB quantity, $\boldsymbol{A}_{a}$ is an edge quantity, and $\boldsymbol{B}_{a}$ is a node quantity. The label $\boldsymbol{F}_{a}$ refers to PDE $a$ being considered, as there are multiple simultaneously coupled PDEs in semiconductor simulation ${ }^{1}$.

2) Node Assembly: Figure 1 shows a mesh node in $2 \mathrm{D}$ surrounded by triangular elements. The $\boldsymbol{B}_{a}$ term is integrated over the NodeVolume. The RHS entry for this scalar quantity is then:

$$
F_{a_{i}}+=B_{a_{i}} \cdot \text { NodeVolume }_{i}
$$

for each node $i$ on the simulation mesh. In this, and subsequent equations, $+=$ represents addition of the term to an existing matrix or vector entry. Similarly, $-=$ represents subtraction.

For the Jacobian:

$$
J_{a_{i}, x_{j}}+=\frac{\partial B_{a_{i}}}{\partial x_{j}} \cdot \text { NodeVolume }_{i}
$$

where $x_{i}$ is a simulation variable on the same node. The Jacobian entry is placed in the row corresponding to equation $a$ and the column corresponding to simulation variable $x$.

3) Edge Assembly : Figure 2 shows the EdgeCouple over which flux is integrated for the $\boldsymbol{A}_{a}$ term in (2). The calculation of EdgeCouple is described in Section II-A.4.

For each edge between adjacent nodes $\langle i, j\rangle$

$$
\begin{aligned}
& F_{a_{i}}+=A_{a_{i, j}} \cdot \text { EdgeCouple }_{i, j} \\
& F_{a_{j}}-=A_{a_{i, j}} \cdot \text { EdgeCouple }_{i, j}
\end{aligned}
$$

where $A_{a_{i, j}}$ is the vector quantity evaluated on a mesh edge, and EdgeCouple $e_{i, j}$ is the cross section of the mesh edge.

\footnotetext{
${ }^{1}$ This paper does not consider our approach to time integration methods or boundary conditions.
}

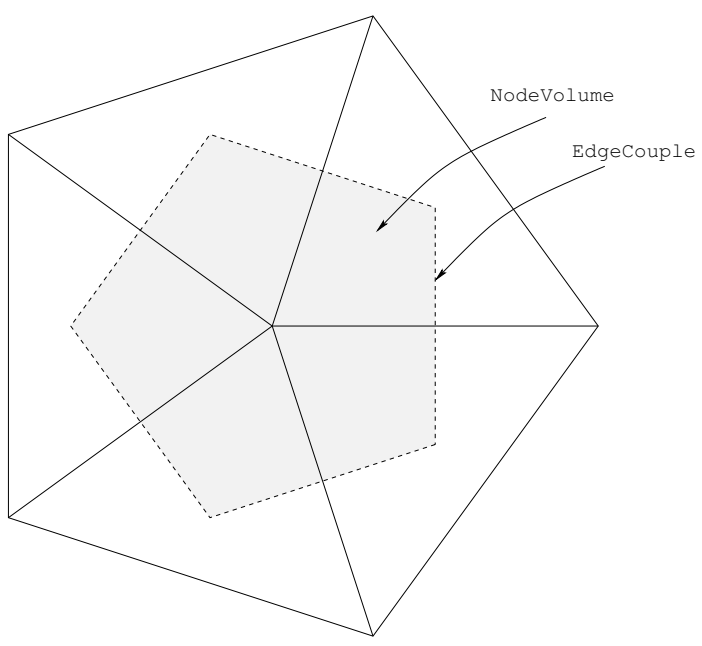

Fig. 1. The 2D mesh cell with an arbitrary number of triangle elements connected to node. The volume of a node is bounded by the perpendicular bisectors of each triangle [20].

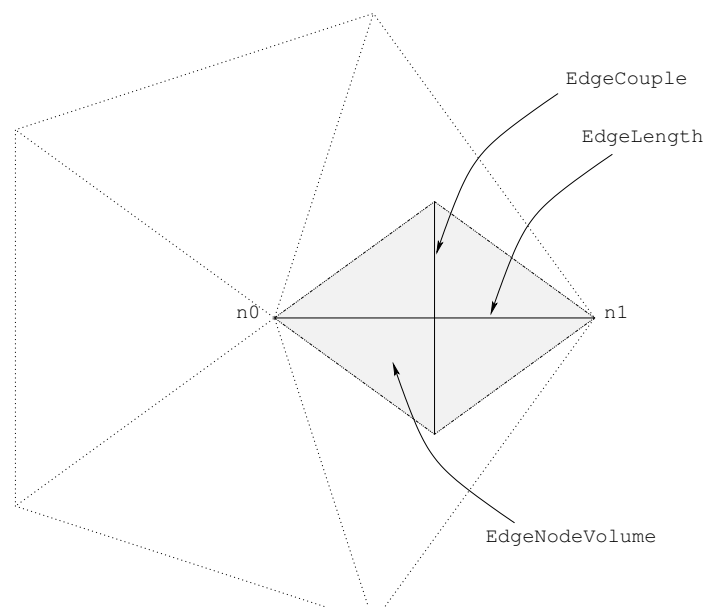

Fig. 2. The 2D mesh cell depicting how flux is integrated along each edge [20]. The EdgeCouple is the length of the perpendicular bisectors for the 2 triangles along the edge connecting nodes $\langle\mathrm{n} 0, \mathrm{n} 1\rangle$.

The Jacobian entries are:

$$
\begin{aligned}
& J_{a_{i}, x_{k}}+=\frac{\partial A_{a_{i, j}}}{\partial x_{k}} \cdot \text { EdgeCouple }_{i, j} \\
& J_{a_{j}, x_{k}}-=\frac{\partial A_{a_{i, j}}}{\partial x_{k}} \cdot \text { EdgeCouple }_{i, j}
\end{aligned}
$$

where $x_{k}$ is the simulation variable on one of the two nodes on the edge.

4) Element Edge Assembly: We consider the $\boldsymbol{C}_{a}$ contribution to (2), which is EEB, and is dependent on variables on all element nodes.

Figure 3 shows a 2D representation of the volume and area over which the integration occurs. In $2 \mathrm{D}$, the ElementEdgeCouple on each edge is from the triangle circumcenter to the center of the edge. Figure 4 shows the element edge volume in 3D, where the ElementEdgeCouple is from the circumcenter of the tetrahedral element, to the circumcenters of the element triangles connected to the element edge, to the center of the element edge. In both $2 \mathrm{D}$ and $3 \mathrm{D}$, the EdgeCouple in Section II-A.3 is calculated by summing 


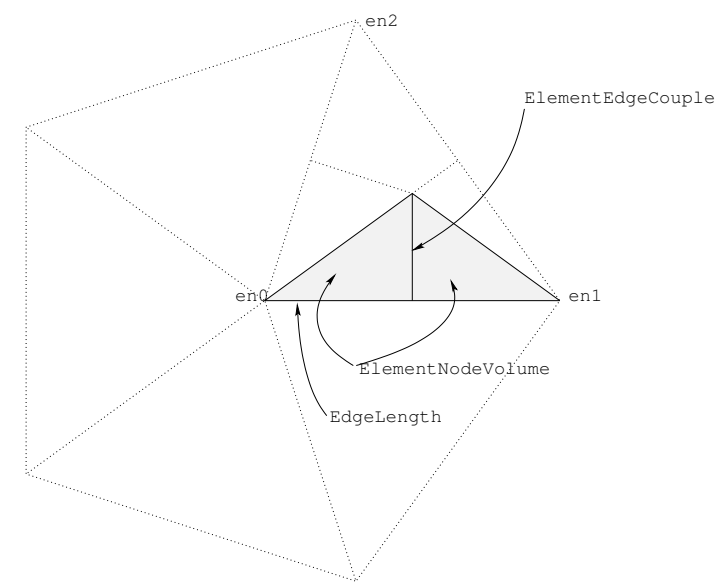

Fig. 3. The mesh cell in 2D. Each triangle is subdivided into 3 sub volumes. The labels for nodes $\langle$ en 0, en 1 , en 2$\rangle$ are specific to the element edge in the sub element bounded by the solid line [20].

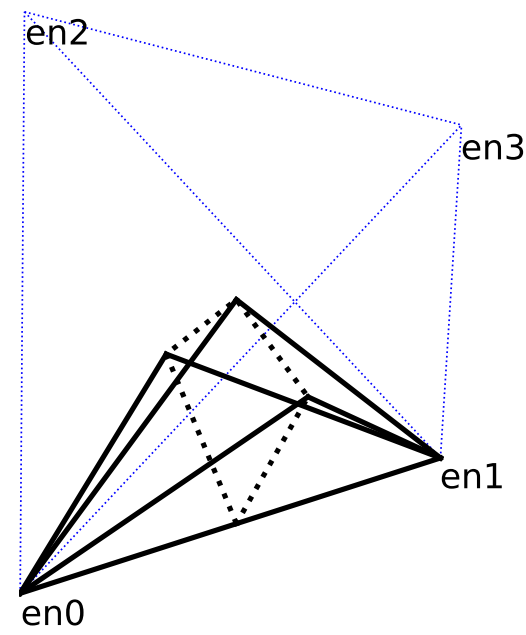

Fig. 4. The element cell in 3D. The tetrahedron is subdivided into 6 subvolumes along each edge. The element edge of interest has the nodes labeled $\langle$ en 0, en 1$\rangle$. The other nodes are labeled $\langle$ en2, en 3$\rangle$.

the ElementEdgeCouple for all elements connected to the edge being considered.

Similar to (5)-(6), the RHS contribution for $\boldsymbol{C}_{a}$ is:

$$
\begin{aligned}
& F_{a_{i}}+=C_{a_{i, j}}^{t} \cdot \text { ElementEdgeCouple } e_{i, j}^{t} \\
& F_{a_{j}}==C_{a_{i, j}}^{t} \cdot \text { ElementEdgeCoupl } \mathrm{e}_{i, j}^{t}
\end{aligned}
$$

where $t$ is the index of the element containing the edge.

Similar to (7)-(8), the Jacobian entries are:

$$
\begin{aligned}
& J_{a_{i}, x_{k}}+=\frac{\partial C_{a_{i, j}}^{t} \cdot \text { ElementEdgeCouple }_{i, j}^{t}}{\partial x_{k}} \\
& J_{a_{j}, x_{k}}-=\frac{\partial C_{a_{i, j}}^{t} \cdot \text { ElementEdgeCouple }_{i, j}^{t}}{\partial x_{k}}
\end{aligned}
$$

where $x_{k}$ is a simulation variable on one of the nodes on the element. In 2D, this is the 2 nodes, $\langle$ en 0 , en 1$\rangle$, on the element edge being considered, and a third node, en2, on a triangular element, as shown in Fig. 3. In 3D, there are 2 nodes on each element edge, $\langle e n 0$, en 1$\rangle$, as well as 2 additional nodes on the tetrahedral element $\langle$ en2, en3 $\rangle$, as shown in Fig. 4.
5) Edge Volume Assembly : It is also possible to do a volume integration using either the edge based or EEB assembly. This is useful for models such as the density gradient model [21] or impact ionization model [15], where contributions are summed to both nodes on an edge. In (5)-(8), the EdgeNodeVolume in Fig. 2 is substituted for EdgeCouple and the assembly is only done with the $+=$ operation. Similarly, for EEB assembly, the ElementNodeVolume in Fig. 3 is substituted for ElementEdgeCouple in (9)-(12).

\section{B. Element Edge Based Fields}

1) Calculating the Vector Field: To calculate a vector field on an edge of a tetrahedral element, we begin by calculating the components along each mesh edge in the device region. For the case of electric field, this is

$$
E_{i, j}=\left(\psi_{i}-\psi_{j}\right) / \text { EdgeLength }_{i, j}
$$

where $\psi_{i}$ and $\psi_{j}$ are the potentials at nodes $\langle i, j\rangle$, and EdgeLength ${ }_{i, j}$ is the distance between them, as shown in Fig. 2. The derivatives with respect to the node potentials are:

$$
\frac{\partial E_{i, j}}{\partial \psi_{i}}=1 / \text { EdgeLength }_{i, j} \quad \frac{\partial E_{i, j}}{\partial \psi_{j}}=-1 / \text { EdgeLength }_{i, j}
$$

In Fig. 5, we show how these scalar fields are used to calculate $\boldsymbol{E}_{t_{0}}$, the vector field based on all edges connect to node 0 . The electric field on each edge is assumed to be

$$
E_{i, j}=\boldsymbol{s}_{i, j} \cdot \boldsymbol{E}_{t_{0}}
$$

where $\boldsymbol{E}_{t_{0}}$ is the field over the entire element, and $\boldsymbol{s}_{i, j}$ is the unit vector along the edge connecting nodes $\langle i, j\rangle$.

For tetrahedra with nodes $\langle 0,1,2,3\rangle$, we use the $x, y, z$ components of the unit vectors to calculate the electric field for all edges connected to node 0

$$
\left(\begin{array}{lll}
s_{0,1}^{x} & s_{0,1}^{y} & s_{0,1}^{z} \\
s_{0,2}^{x} & s_{0,2}^{y} & s_{0,2}^{z} \\
s_{0,3}^{x} & s_{0,3}^{y} & s_{0,3}^{z}
\end{array}\right)\left(\begin{array}{l}
E_{t_{0}}^{x} \\
E_{t_{0}}^{y} \\
E_{t_{0}}^{z}
\end{array}\right)=\left(\begin{array}{l}
E_{0,1} \\
E_{0,2} \\
E_{0,3}
\end{array}\right)
$$

which can be written more compactly as

$$
\mathbf{S}_{t_{0}} \cdot \boldsymbol{E}_{t_{0}}=\left(\begin{array}{lll}
E_{0,1} & E_{0,2} & E_{0,3}
\end{array}\right)^{T}
$$

so that $\boldsymbol{E}_{t_{0}}$ is found by solving this small linear system.

The derivatives with respect to the node potentials are then

$$
\begin{aligned}
\mathbf{S}_{t_{0}} \cdot \frac{\partial \boldsymbol{E}_{t_{0}}}{\partial \psi_{0}} & =\left(\begin{array}{lll}
\frac{\partial E_{0,1}}{\partial \psi_{0}} & \frac{\partial E_{0,2}}{\partial \psi_{0}} & \frac{\partial E_{0,3}}{\partial \psi_{0}}
\end{array}\right)^{T} \\
\mathbf{S}_{t_{0}} \cdot \frac{\partial \boldsymbol{E}_{t_{0}}}{\partial \psi_{1}} & =\left(\begin{array}{lll}
\frac{\partial E_{0,1}}{\partial \psi_{1}} & 0 & 0
\end{array}\right)^{T} \\
\mathbf{S}_{t_{0}} \cdot \frac{\partial \boldsymbol{E}_{t_{0}}}{\partial \psi_{2}} & =\left(\begin{array}{lll}
0 & \frac{\partial E_{0,2}}{\partial \psi_{2}} & 0
\end{array}\right)^{T} \\
\mathbf{S}_{t_{0}} \cdot \frac{\partial \boldsymbol{E}_{t_{0}}}{\partial \psi_{3}} & =\left(\begin{array}{lll}
0 & 0 & \frac{\partial E_{0,3}}{\partial \psi_{3}}
\end{array}\right)^{T}
\end{aligned}
$$

In $3 \mathrm{D}$, we treat the field for the edge between nodes $\langle 0,1\rangle$ using an average:

$$
\boldsymbol{E}_{t_{0,1}}=0.5 \cdot\left(\boldsymbol{E}_{t_{0}}+\boldsymbol{E}_{t_{1}}\right)
$$




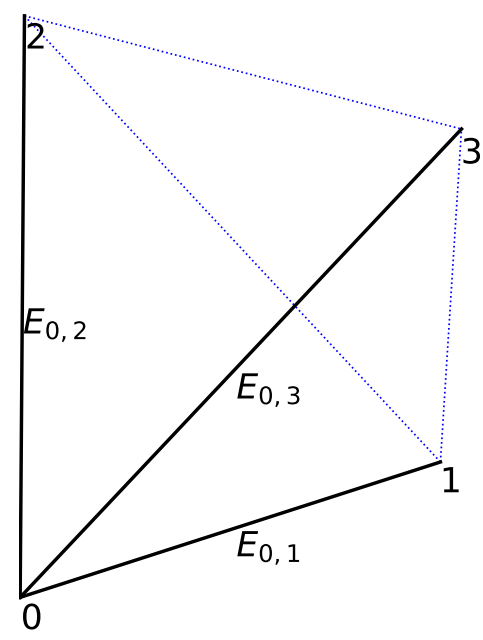

Fig. 5. Depiction on how vector field is calculated from the scalar fields calculated along the $\mathbf{3}$ edges connected to node $\mathbf{0}$.

where $\boldsymbol{E}_{t_{1}}$ is calculated by considering node 1 as the node of interest in (16)-(21) ${ }^{2}$.

The derivative with respect to the node potential is then:

$$
\frac{\partial \boldsymbol{E}_{t_{0,1}}}{\partial \psi_{k}}=0.5 \cdot\left(\frac{\partial \boldsymbol{E}_{t_{0}}}{\partial \psi_{k}}+\frac{\partial \boldsymbol{E}_{t_{1}}}{\partial \psi_{k}}\right)
$$

where $k$ refers to one of the 4 nodes on the element.

We assume that the average employed in (22)-(23) is appropriate for creating vectors from edge based models. It can be shown for electric field that $\boldsymbol{E}_{t_{0}}=\boldsymbol{E}_{t_{1}}$, so the average is valid. For cases like current density, which does not have this property, this assumption appears valid for the simulations in Section III-C.

Once the vector fields are attained, they are used to calculate the components for the element edge assembly in (9)-(12), by first converting to a scalar value, and then adding to $\mathbf{J}$ and $\boldsymbol{F}$. Scalar models defined as an edge or element edge model, can also be used in calculations with the vector field components. This will be described in Section II-C, where the approach is described for the polarization vector in ferroelectric materials, as well as for the transverse electric field used for mobility models.

2) Computation: The $\boldsymbol{S}_{t_{0}}$ term in (17) is calculated once for each node of each element and is assigned as $\boldsymbol{S}_{t_{0}}$ or $\boldsymbol{S}_{t_{1}}$, based on the element edge of interest. The element field is computed for each Newton iteration (see (1)).

3) Limitations on Element Type: We only consider triangular elements in $2 \mathrm{D}$ and tetrahedral elements in $3 \mathrm{D}$. In $3 \mathrm{D}$, we require the centers of the tetrahedra circumspheres are inside the element, so that the volume and surface integrals are calculated correctly [2]. In 2D, the circumcenter of triangular elements should be inside the circumcircle [1]. Other FVM TCAD simulators may have less stringent restrictions.

4) Derivative Accuracy: Good convergence behavior is often dependent on having accurate derivatives for the simulation

\footnotetext{
${ }^{2}$ In 2D, we use the edge average from [10], [15], based on a weighting of the length of perpendicular bisectors of the triangular elements.
}

models [1]. We demonstrate this with the simulation example in Section III-A. Accurate derivatives are also important for small-signal analysis, and for the impedance field method [22]-[24].

\section{Generalized PDE Approach}

In DEVSIM, models are implemented as symbolic expressions, and they are assembled and solved [17], [20]. Symbolic differentiation is employed to get the required derivatives. The simulator accepts equations in the form of:

Node models on nodes (Sec. II-A.2)

Edge models on edges (Sec. II-A.3)

Element edge models on element edges (Sec. II-A.4)

The GPDE equations are input by calling commands from a Python script [19]. The simulator is a module loaded by the interpreter.

To evaluate a vector model, we start with an edge model $\mathrm{F}$ and its derivatives with respect to a node variable psi at nodes $\langle 0,1\rangle$

F, F:psi@n $0, \quad F: p s i @ n 1$

where $\mathrm{F}$ is defined using a symbolic expression like (13), and the derivatives using expressions like (14).

The element_from_edge_model command is used to create element edge models as:

F_X, F_Y, F_z

for each vector component using (22) and the derivatives

F_x:psi@en0, F_y:psiden0, F_z:psi@en0

F_x:psiden1, F_y:psiden1, F_z:psiden1

F_x:psiden2, F_y:psiden2, F_z:psiden2

F_x:psiden3, F_y:psiden3, F_z:psiden3

using (23). The first term represents the derivative of $\mathrm{F}_{-} \mathrm{x}$ with respect psi at node 0 , and the other terms follow in a similar fashion.

During assembly, we use the conventions for $\langle$ en0, en1, en2, en3〉 shown in Fig. 4. Similar transformations are available to transform node models to edge models (e.g. (13)). For element edge model expressions, scalar edge models are implicitly transformed to element edge models, but with the derivatives with respect to $\langle e n 2$, en 3$\rangle$ set equal to zero.

1) Ferroelectric Model: A ferroelectric insulator model may be implemented using scripting. The Poisson equation is:

$$
\nabla \cdot(\varepsilon \boldsymbol{E}+\boldsymbol{P})=0
$$

where the polarization, $\boldsymbol{P}$, is a nonlinear function of $\boldsymbol{E}$ and $\varepsilon$ is the permittivity.

We employ an empirical equation from [12], [25], [26] to calculate each component of $\boldsymbol{P}$. The polarization along each axis $i \in(x, y, z)$ reads:

$$
P_{i}=P_{S} c_{i} \tanh \left[\omega\left(E_{i}-\mathcal{E}_{F_{C}}\right)\right]+P_{S} c_{o f f}
$$

where

$$
\omega=\ln \left(\frac{P_{S}+P_{r}}{P_{S}-P_{r}}\right) /\left(2 \mathcal{E}_{F_{C}}\right)
$$

$E_{i}$ is the $i$ component of the electric field, $P_{S}$ is the saturation polarization, $P_{r}$ is the remnant polarization, and $\mathcal{E}_{F_{C}}$ is the 
coercive field. The parameters $c_{o f f} \in[-1,1]$ and $c_{i} \in(0,1]$ account for the polarization hysteresis in each direction.

We evaluate (24) on each element edge as

$$
\begin{array}{r}
\left(P_{t_{0,1}}^{x}+\varepsilon E_{t_{0,1}}^{x}\right) s_{0,1}^{x}+\left(P_{t_{0,1}}^{y}+\varepsilon E_{t_{0,1}}^{y}\right) s_{0,1}^{y} \\
+\left(P_{t_{0,1}}^{z}+\varepsilon E_{t_{0,1}}^{z}\right) s_{0,1}^{z}
\end{array}
$$

where $P_{t_{0,1}}^{x}, P_{t_{0,1}}^{y}$, and $P_{t_{0,1}}^{z}$ are evaluated from (25)-(26) for each element edge using (9)-(10). Derivatives of this expression are also included using (11)-(12).

2) Field Dependent Mobility: To calculate the mobility for the simulations in Section III-C, we first calculate the electric field parallel to current flow as:

$$
E_{t_{0,1}}^{\|}=\boldsymbol{j}_{t_{0,1}} \cdot \boldsymbol{E}_{t_{0,1}} /\left|\boldsymbol{j}_{t_{0,1}}\right|
$$

where $j_{t_{0,1}}$ is the current density vector calculated using

$$
\mathbf{S}_{t_{0}} \cdot \boldsymbol{j}_{t_{0}}=\left(\begin{array}{lll}
j_{0,1} & j_{0,2} & j_{0,3}
\end{array}\right)^{T}
$$

where $j_{0,1}, j_{0,2}$, and $j_{0,3}$ are edge current densities using a suitable low field mobility, and then averaged onto the element edge using the same average as (22). The vector $\boldsymbol{j}_{t_{0,1}}$ is used to find the direction of current flow, and is not the final current density. The transverse electric field is then calculated using:

$$
\left|E_{t_{0,1}}^{\perp}\right|=\sqrt{\left|\boldsymbol{E}_{t_{0,1}}\right|^{2}-\left|E^{\|_{t_{0,1}}}\right|^{2}}
$$

We implement the low field mobility from [27] using:

$$
\frac{1}{\mu_{\mathrm{lf}}}=\frac{1}{\mu_{\mathrm{b}}}+\frac{1}{\mu_{\mathrm{ac}}}+\frac{1}{\mu_{\mathrm{sr}}}
$$

where $\mu_{\mathrm{b}}$ is a carrier concentration, doping dependent, and temperature dependent bulk mobility model. The surface mobility terms for acoustic phonon scattering $\left(\mu_{\mathrm{ac}}\right)$ and surface roughness $\left(\mu_{\mathrm{sr}}\right)$ are both dependent on $E_{t_{0,1}}^{\perp}$ and are also dependent on carrier concentration, doping, and temperature.

First, $\mu_{\mathrm{b}}$ is calculated on the node, and then it and its derivatives are averaged onto each edge. Being vector field dependent, $\mu_{\mathrm{ac}}$ and $\mu_{\mathrm{sr}}$ are calculated by averaging nodal quantities onto the element edge and evaluating the expressions with $E_{t_{0,1}}^{\perp}$.

The velocity saturated mobility is then calculated using

$$
\mu_{t_{0,1}}^{\mathrm{hf}}=\frac{2 \mu_{t_{0,1}}^{\mathrm{lf}}}{1+\left(1+4\left(\frac{\mu_{t_{0,1}}^{\mathrm{lf}} F_{0,1}^{\mathrm{hfs}}}{v_{s}}\right)^{2}\right)^{\frac{1}{2}}}
$$

where $v_{s}$ is the saturation velocity and the driving force is

$$
F_{0,1}^{\mathrm{hfs}}=\frac{1}{q} \nabla E_{F_{n}}
$$

is the gradient of the quasi-Fermi level [28] along the edge. We chose (33) over the electric field parallel to current flow used in [27], as it overcomes difficulties when the electric field is in the opposite direction of current flow [1]. The EEB current densities are evaluated and then integrated into the continuity equations using (9)-(12).

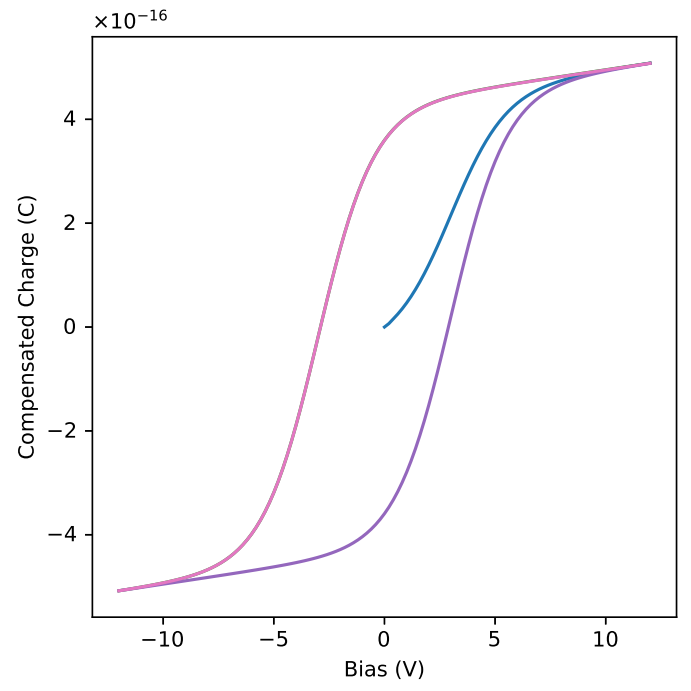

Fig. 6. The ferro capacitor 3D simulation.

\section{SimULATION EXAMPLES}

\section{A. Ferrocapacitor Simulation}

TetGen [29] was used to generate a tetrahedral mesh of a cube and contacts on the top and bottom. Not all of the elements met our requirements mentioned in Section II-B.3, and this resulted in a discrepancy between the 3D result and a similar 2D structure. A script was written to mark bad elements for refinement and to create a background mesh to load back into TetGen. A few iterations between the mesher and the script resulted a 3D simulation with good agreement to the $2 \mathrm{D}$ results.

The mesh and physics are loaded via scripts into the simulator, implementing the insulator model from Sec. IIC.1. Fig. 6 shows the compensated charge versus the applied voltage for a ferroelectric capacitor. From the initial zero bias condition, the voltage is ramped over the range between $\pm 15 \mathrm{~V}$. The hysteresis in the charge due to the polarization is apparent in the figure.

As a test of the accuracy of our approach, we repeated the simulations with derivative terms with respect to nodes $\langle e n 2$, en3 $\rangle$ set to 0 . This emulates the condition in GPDE simulators which evaluate vector effects over all adjacent nodes, but may restrict their derivative information on edges [4], [14].

The primary convergence criteria in our simulator is the relative update norm, which is calculated at each node $i$ as:

$$
r_{i}^{m}=\frac{\left|\psi_{i}^{m}-\psi_{i}^{m-1}\right|}{\left|\psi_{i}^{m-1}\right|+10^{-10}}
$$

where $m$ is the iteration number, and $\psi_{i}^{m}$ is the new solution value after the update. The norm is taken at the node with the largest value of $r$.

Fig. 7 shows the relative update norm versus iteration number for one of the bias points. With all derivatives accounted for, it takes 2 iterations to get a relative update norm near $10^{-15}$, which is the limit of accuracy for double precision floating point arithmetic. This number of iterations is expected, since the equation derivatives are constant with respect to bias. 


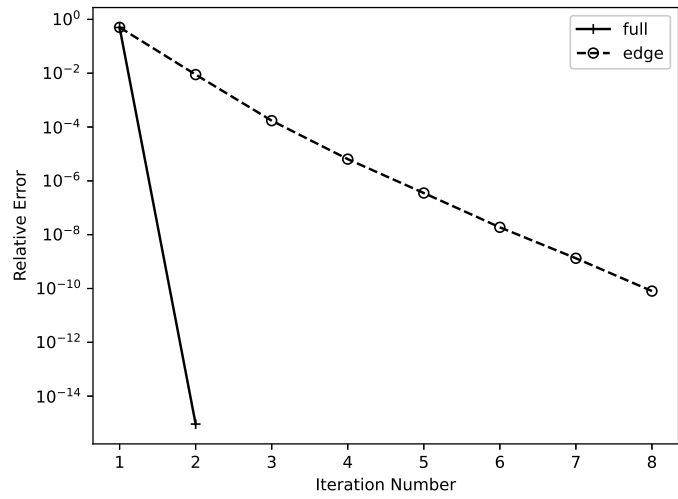

Fig. 7. The relative update norm versus iteration number. The solid line is for derivatives for all nodes on the element. The dashed line is when only nodes on the edge are considered.

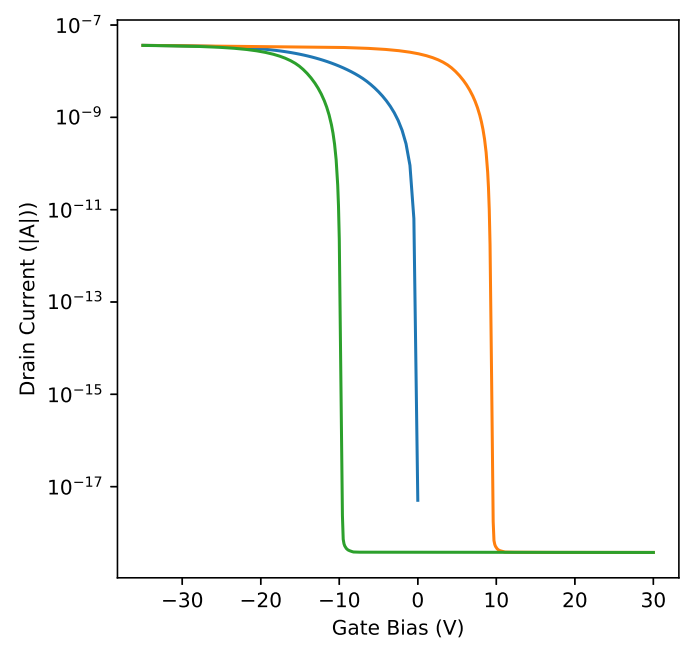

Fig. 8. The p-type FeFET 2D simulation.

When the off edge derivatives are neglected, the iteration count is higher to to attain a relative update norm within $10^{-10}$.

\section{B. FeFET Simulation}

We simulated a 2D p-type FeFET with a top contact/bottom gate architecture. We solved for the ferroelectric insulator using (24). Ohmic contacts were set at the source and drain electrodes and the standard DDM equations were solved in the semiconductor. A triangular mesh was generated using Gmsh [30]. Extended precision math mode was enabled in the equation assembly to prevent numerical noise in the off state current results. Fig. 8 shows the drain current versus gate voltage. The simulation details and an analysis of the switching processes of FeFET with respect to its structure is presented in [31].

It is important to note that when the derivative off the edge on node en2 was set to 0 , the simulation failed before the sweep of biases could finish. Therefore, the full set of derivatives using the EEB approach was necessary to run the simulations. This is consistent with the findings in [14] concerning the effects of incomplete derivatives on the convergence of ferroelectric device simulations.

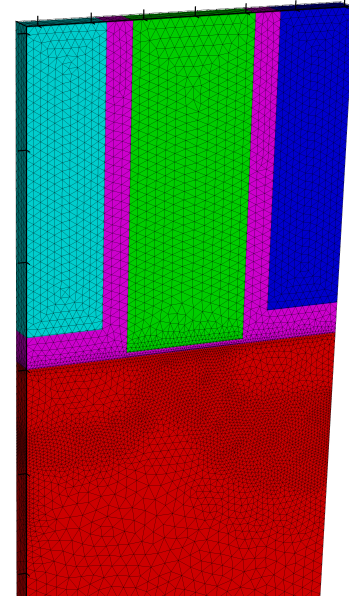

Fig. 9. The $90 \mathrm{~nm}$ 3D MOSFET. The polysilicon gate (2) is surrounded by oxide (5) and two nitride regions $(3,4)$. The bulk region (1) has a 120 $\mathrm{nm}$ drawn gate length. The source and drain contacts are both $50 \mathrm{~nm}$ underneath the nitride regions. A body contact was placed on the bottom of the $60 \mathrm{~nm}$ silicon region. The oxide thickness is $4.9 \mathrm{~nm}$ and the device is $25 \mathrm{~nm}$ thick.

\section{MOSFET Simulation}

We simulated a 3D MOSFET, based on a doping profile and 2D structure for a $90 \mathrm{~nm}$ n-channel MOSFET archived in [32]. For the simulations, we created a 3D mesh using Cubit [33]. A simulation based mesh refinement stategy was employed to achieve the final mesh, with a mesh sizing field collected over a range of biases. This results in the asymmetry of the mesh elements sizes for the mesh shown in Fig. 9.

Using the scripting interface, we implemented the standard DDM for Poisson, electron-continuity, and hole-continuity equations, with SRH recombination [1]. We used the field dependent mobility model described in II-C.2 using the default physical model parameters from [27], without any additional calibration.

We compared the mobility models using these three cases:

- Bulk mobility model $\left(\mu=\mu_{b}\right)$

- Bulk mobility with velocity saturation $\left(\mu=\mu_{\mathrm{hf}}\right.$ with $\mu_{\mathrm{lf}}=\mu_{\mathrm{b}}$ in (32))

- Full mobility model with velocity saturation $\left(\mu=\mu_{\mathrm{hf}}\right)$

where the normal electric field for surface mobility was implemented using (30).

Figure 10 shows $I_{D}$ versus $V_{G S}$ for the three cases. The $I_{D}$ versus $V_{G S}$ dependence is shown in Fig. 11. Consistent with the theory, the $E^{\perp}$ dependent mobility results in a lowering of the drain current.

\section{ACKNOWLEDGMENT}

J. E. Sanchez thanks E. J. Chiles, M. E. Sanchez, and C. M. Gotwalt for their assistance in the editing of the manuscript. The authors thank the anonymous reviewers for their feedback.

\section{REFERENCES}

[1] S. Selberherr, Analysis and simulation of semiconductor devices. NY: Springer-Verlag, 1984.

[2] M. R. Pinto, "Comprehensive semiconductor device simulation for silicon ULSI," Ph.D. dissertation, Stanford University, 1990. 


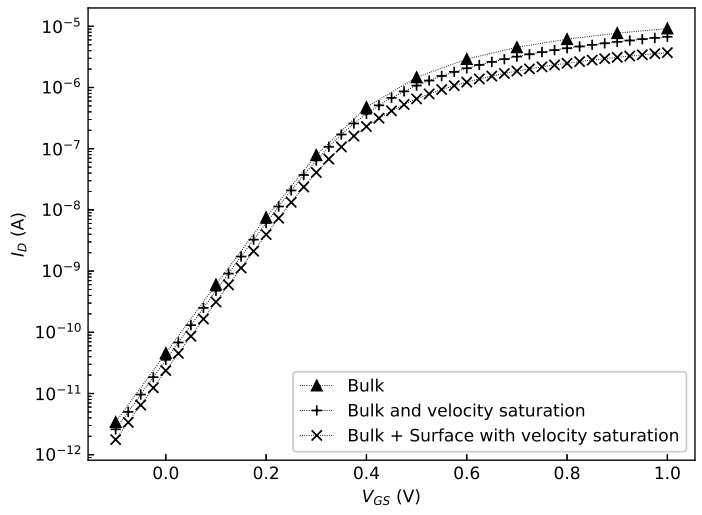

Fig. 10. Drain current, $\boldsymbol{I}_{\boldsymbol{D}}$, versus $\boldsymbol{V}_{G S}$ for $\boldsymbol{V}_{\boldsymbol{D} S}=0.1 \mathrm{~V}$ and $\boldsymbol{V}_{\boldsymbol{S B}}=0$.

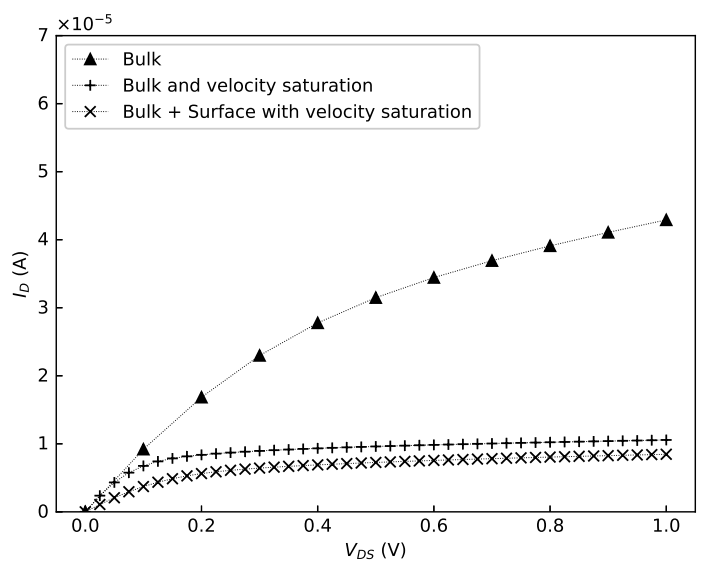

Fig. 11. Drain current, $\boldsymbol{I}_{D}$, versus $\boldsymbol{V}_{D S}$ for $\boldsymbol{V}_{G S}=1 \mathrm{~V}$. and $\boldsymbol{V}_{S B}=0$.

[3] D. L. Scharfetter and H. K. Gummel, "Large-signal analysis of a silicon Read diode oscillator," IEEE Trans Electron Devices, vol. ED-16, no. 1, pp. 64-77, Jan. 1969, 10.1109/T-ED.1969.16566.

[4] K. M. Kramer and W. N. G. Hitchon, Semiconductor Devices: A Simulation Approach. Up Saddle River, NJ: Prentice Hall PTR, 1997.

[5] D. J. Cummings, M. E. Law, S. Cea, and T. Linton, "Comparison of discretization methods for device simulation," in 2009 Int Conf SISPAD, 2009, pp. 1-4, doi: 10.1109/SISPAD.2009.5290236.

[6] E. Patrick, N. Rowsey, and M. E. Law, "Total dose radiation damage: A simulation framework," IEEE Trans Nucl Sci, vol. 62, no. 4, pp. 16501657, 2015, doi: 10.1109/TNS.2015.2425226.

[7] P. Bochev, K. Peterson, and X. Gao, "A new control volume finite element method for the stable and accurate solution of the drift-diffusion equations on general unstructured grids," Comput Methods Appl Mech Eng, vol. 254, pp. 126-145, 02 2013, doi: 10.1016/j.cma.2012.10.009.

[8] COMSOL, "Analyze semiconductor devices at the fundamental level with the semiconductor module," last accessed 09/15/2020. [Online]. Available: https://www.comsol.com/semiconductor-module

[9] L. Chen and H. Bagci, "Steady-state simulation of semiconductor devices using discontinuous Galerkin methods," IEEE Access, vol. 8, pp. 16203-16215, 2020, doi: 10.1109/ACCESS.2020.2967125.

[10] S. E. Laux and R. G. Byrnes, "Semiconductor device simulation using generalized mobility models," IBM J. Res. Dev., vol. 29, no. 3, pp. 289301, May 1985, doi: 10.1147/rd.293.0289.

[11] S. L. Miller, R. D. Nasby, J. R. Schwank, M. S. Rodgers, and P. V. Dressendorfer, "Device modeling of ferroelectric capacitors," J Appl Phys, vol. 68, no. 12, pp. 6463-6471, 1990, doi: 10.1063/1.346845.

[12] M. Ghittorelli, T. Lenz, H. Sharifi Dehsari, D. Zhao, K. Asadi, P. W. M. Blom, Z. M. Kovács-Vajna, D. M. de Leeuw, and F. Torricelli, "Quantum tunnelling and charge accumulation in organic ferroelectric memory diodes," Nat Commun, vol. 8, no. 1, p. 15741, 2017, doi: 10.1038/ncomms 15841 .

[13] Z. Yu, D. W. Yergeau, and R. W. Dutton, "Algorithm for evaluating nodal vector quantities in device simulation and its applications to modeling quantum mechanical effects in sub-50nm MOSFETs," in Int Symp VLSI Tech, Syst App, 2003, pp. 261-264, doi: 10.1109/VTSA.2003.1252603.

[14] T. Ikegami, K. Fukuda, and J. Hattori, "Implementation of automatic differentiation to Python-based semiconductor device simulator," in 2019 Int Conf SISPAD, 2019, pp. 1-4, doi: 10.1109/SISPAD.2019.8870377.

[15] S. E. Laux and B. M. Grossman, "A general control-volume formulation for modeling impact ionization in semiconductor transport," IEEE Trans Electron Devices, vol. 32, no. 10, pp. 2076-2082, 1985, doi: 10.1109/T-ED.1985.22241.

[16] O. Triebl and T. Grasser, "Investigation of vector discretization schemes for box volume methods," in Tech Proc 2007 NSTI Nanotechnology Conf and Trade Show, Vol 3, 2007, pp. 61-64.

[17] J. E. Sanchez, "Semiconductor device simulation using DEVSIM," in Open Source TCAD/EDA for Compact Modeling, W. Grabinski and D. Tomaszewski, Eds. New York: Springer, 2021.

[18] DEVSIM LLC, "DEVSIM TCAD semiconductor device simulator," Available: https://devsim.org.

[19] Python Software Foundation, "Python," https://python.org

[20] DEVSIM LLC, "DEVSIM manual," Available: https://devsim.net, doi: $10.5281 /$ zenodo. 4583208 .

[21] A. Wettstein, O. Penzin, and E. Lyumkis, "Integration of the density gradient model into a general purpose device simulator," VLSI Design, vol. 15, no. 4, pp. 751-759, 2002, doi: 10.1080/1065514021000012363.

[22] S. E. Laux, "Techniques for small-signal analysis of semiconductor devices," IEEE Trans Electron Devices, vol. 32, no. 10, pp. 2028-2037, 1985, doi: 10.1109/T-ED.1985.22235.

[23] F. Bonani, G. Ghione, M. R. Pinto, and R. K. Smith, "An efficient approach to noise analysis through multidimensional physics-based models," IEEE Trans Electron Devices, vol. 45, no. 1, pp. 261-269, 1998, doi: 10.1109/16.658840.

[24] K. El Sayed, A. Wettstein, S. D. Simeonov, E. Lyumkis, and B. Polsky, "Investigation of the statistical variability of static noise margins of SRAM cells using the statistical impedance field method," IEEE Transactions on Electron Devices, vol. 59, no. 6, pp. 1738-1744, 2012, doi: 10.1109/TED.2012.2189860.

[25] S. L. Miller and P. J. McWhorter, "Physics of the ferroelectric nonvolatile memory field effect transistor," J Appl Phys, vol. 72, no. 12, pp. 5999-6010, 1992, doi: 10.1063/1.351910.

[26] B. Shrestha, R. Pieper, W. Wondmagegn, and N. Satyala, "Modeling and characterization for polarization hysteresis of ferroelectric polymers," in 2011 Proceedings of IEEE Southeastcon, Conference Proceedings, pp. 224-227, 10.1109/SECON.2011.5752938.

[27] M. N. Darwish, J. L. Lentz, M. R. Pinto, P. M. Zeitzoff, T. J. Krutsick, and H. H. Vuong, "An improved electron and hole mobility model for general purpose device simulation," IEEE Trans Electron Devices, vol. 44, no. 9, pp. 1529-1538, 1997, doi: 10.1109/16.622611.

[28] R. Pierret, Advanced Semiconductor Fundamentals, 2nd ed. Upper Saddle River, NJ: Pearson, 2003

[29] H. Si, "TetGen, a Delaunay-based quality tetrahedral mesh generator," ACM Trans. Math. Softw., vol. 41, no. 2, Feb. 2015, doi: $10.1145 / 2629697$.

[30] C. Geuzaine and J.-F. Remacle, "Gmsh: a three-dimensional finite element mesh generator with built-in pre- and post-processing facilities," Int J Numer Methods Eng, vol. 79, pp. 1309-1331, 2009, doi: 10.1002/nme.2579.

[31] Q. Chen, D. Lin, Q. Wang, J. Yang, J. Sanchez, and G. Zhu, "TCAD simulation on the impact of source and drain contact positions in thin film ferroelectric transistors," submitted for publication.

[32] D. A. Antoniadis, I. J. Djomehri, K. M. Jackson, and S. Miller, "Well-Tempered bulk-Si NMOSFET device home page," Nov. 2001, doi: 10.5281/zenodo.4672795.

[33] Coreform LLC, "Coreform Cubit (Version 2021.3)," Available: https://coreform.com. 